\title{
El prometedor futuro del análisis de clase: Una respuesta a las críticas recientes
}

\section{[The promising future of class analysis: A response to recent critiques]}

\author{
John H. Goldthorpe \& Gordon Marshall
}

\begin{abstract}
Resumen
El análisis de clase ha sido criticado recientemente desde varios puntos de vista. En este artículo sostenemos que mucha de esta crítica está fuera de lugar y que, en cuanto programa de investigación, la promesa del análisis de clase está lejos de agotarse. La primera parte del artículo clarifica la naturaleza y el propósito del análisis de clase, tal y como lo entendemos, y en particular lo distingue del análisis de clase de la sociología marxista. Luego la segunda parte defiende la relevancia actual del análisis de clase, en nuestra concepción de éste, revisando hallazgos de tres áreas centrales de investigación en curso.
\end{abstract}

Palabras clave: análisis de clase, movilidad social, educación, política.

\begin{abstract}
Class analysis has recently been criticised from a variety of standpoints. In this paper we argue that much of this criticism is misplaced and that, as a research programme, the promise of class analysis is far from exhausted. The first part of the paper clarifies the nature and purpose of class analysis, as we would understand it, and in particular distinguishes it from the class analysis of Marxist sociology. The second part then makes the case for the continuing relevance of class analysis, in our conception of it, by reviewing findings from three central areas of current research.
\end{abstract}

Keywords: class analysis, social mobility, education, politics.

Este manuscrito es una traducción al español de $\mathrm{e}^{\mathrm{i}}$ Current manuscript is the Spanish translation of ${ }^{i i}$ :

Goldthorpe, J. H., \& Marshall, G. (1992). The promising future of class analysis: a response to recent critiques.

Sociology, 16(3), 381-400. Copyright (C) 1992 by Sage Publications, Ltd.

\footnotetext{
i La traducción de este manuscrito fue desarrollada por Guillermo Palacios (guillermo.palacios@ug.uchile.cl) de la Universidad de Chile con la autorización de Sage Publications, Ltd. (Lic. 4223730931713). El traductor declara que la traducción se hizo de la manera más fiel posible al original, salvo en los formatos de referencias y citas que fueron adaptados al formato usado por la Revista de Sociología. Aunque se hicieron esfuerzos para asegurar que los contenidos de esta publicación son correctos, ni los autores ni los editores aceptan cualquier responsabilidad y, por lo tanto, expresamente se excluyen en la máxima extensión posible bajo la ley vigente de cualquier responsabilidad respecto de los contenidos de este artículo incluyendo limitaciones, errores, omisiones, imprecisiones o futuras traducciones o cualquier tipo de consecuencias que se deriven de ellas. Nada en esta nota debiese excluir las responsabilidades que no se pueden excluir por ley.

iThe translation of this manuscript was developed by Guillermo Palacios (guillermo.palacios@ug.uchile.cl) from University of Chile with authorization of Sage Publications, Ltd. (Lic. 4223730931713). The translator declares that the translation was made more faithfully to the original. Citations and references were adapted to the style of the Revista de Sociología. While every effort has been made to ensure that the contents of this publication are factually correct, neither the authors nor the publishers accepts, and they thereby expressly exclude to the fullest extent permissible under applicable law, any and all liability arising from the contents published in this Article, including, without limitation, from any errors, omissions, inaccuracies in original or following translation, or for any consequences arising therefrom. Nothing in this notice shall exclude liability which may not be excluded by law.
} 


\section{INTRODUCCIÓN}

¿Cuáles son las perspectivas para el análisis de clase? Recientemente, críticos prominentes quienes escriben desde una variedad de puntos de vista, han descartado esta iniciativa por poco convincente e improductiva. Nuestro propio trabajo ha sido un blanco frecuente de ellos. En el presente artículo, sin embargo, nuestro objetivo inicial no es responder a tales cargos en defensa propia, sino hacer valer el tipo de análisis de clase que nuestro trabajo representa, puesto que afirmamos que su promesa está lejos de agotarse.

El documento incluye dos partes. En la primera, buscamos clarificar la naturaleza y el propósito del análisis de clase tal y como lo entendemos, en particular con el fin de distinguirlo del análisis de clase de la sociología marxista. Esto es necesario porque algunos críticos -incluyendo a Hindess (1987), Holton y Turner (1989) y Sørensen (1991)-, en nuestra opinión, no han hecho esta distinción adecuadamente, mientras otros, muy especialmente Pahl (1989), han fallado por completo al intentarlo. Además, pueden observarse varios casos de autores que, habiendo perdido la fe en el análisis de clase marxista que alguna vez comandó su lealtad, o al menos su simpatía, ahora enfrentan una evidente dificultad en concebir algún otro tipo de enfoque. Gorz (1982), Hobsbawm (1981), Bauman (1982), Lukes (1984), y Offe (1985) son ejemplos obvios de ello.

En la segunda parte del artículo defendemos la relevancia actual del análisis de clase, en nuestra propia concepción de éste, revisando hallazgos de tres áreas centrales de investigación en curso. Aquí buscamos discrepar más específicamente con las aseveraciones hechas por Pahl (1989) según las cuales, en las sociedades modernas, "la clase como concepto está dejando de ser útil para la sociología" (p. 70), y por Holton y Turner (1989) de que estamos ahora "en una situación donde la persistencia del lenguaje de clase se explica más en términos de la figura metafórica de la retórica de clase que de cualquier persuasión intelectual clara" (p. 186).

\section{El análisis de clase en cuanto proyecto de investigación}

El análisis de clase, en nuestra perspectiva, tiene como preocupación central el estudio de las relaciones entre estructuras de clase, movilidad de clase, desigualdades basadas en la clase y acción con base en la clase. Más específicamente, explora las interconexiones entre posiciones definidas por relaciones de empleo en mercados de trabajo y unidades de producción en diferentes sectores de las economías nacionales, los procesos a través de los cuales los individuos y las familias son distribuidos y redistribuidos entre estas posiciones a través del tiempo, y las consecuencias de esto para sus oportunidades de vida y para las identidades sociales que adoptan y los valores sociales e intereses que persiguen. Entendido de esta manera, el análisis de clase no implica un compromiso con ninguna teoría de clases en particular sino, en vez de eso, con un programa de investigación - 
en el sentido de Lakatos (1970)- dentro del cual diferentes teorías que incluso son rivales pueden ser formuladas y luego evaluadas en términos de su rendimiento heurístico y explicativo.

Puede preguntarse, y los críticos en efecto lo han hecho (e.g. Holton \& Turner 1989) por qué tal programa debería ser seguido en primer lugar. Podríamos pensar que la respuesta es lo suficientemente obvia. El programa es atractivo en cuanto representa una forma específica de investigar interconexiones del tipo que siempre han involucrado a la imaginación sociológica: es decir, entre estructuras macrosociales formadas históricamente por un lado, y la experiencia cotidiana de individuos dentro de sus ambientes sociales particulares por otro, junto con los patrones de acción que provienen de esta experiencia. Estas son precisamente el tipo de interconexiones que, en palabras de Wright Mills (1959), le permiten a uno relacionar la biografía a la historia y los "problemas personales" con los "asuntos públicos". Desde un punto de vista analítico, este programa también promete una economía explicativa: la habilidad de usar unos pocos conceptos bien definidos tales como posición de clase, orígenes de clase, movilidad o inmovilidad de clase, para explicar en gran parte lo que pasa o no pasa a los individuos en diferentes aspectos de sus vidas sociales y cómo ellos responden subsecuentemente.

Pero a priori sólo hay atractivo y promesa. Si el programa de investigación del análisis de clase resulta valioso -es decir es progresivo en vez que degenerativo- se debe decidir en base a los resultados que produzca. No suponemos la preeminencia de la clase. Por el contrario, es parte del programa de investigación que se considere también que son o se están volviendo de mayor importancia las teorías que sostienen que las relaciones de clases tienen un menor efecto en las oportunidades de vida y en la acción social o que otras relaciones y atributos definidos, por ejemplo, por ingreso y consumo, status o estilo de vida, etnicidad o género.

Debería ser evidente que el análisis de clase concebido de este modo difiere significativamente del análisis de clase de la sociología marxista. Sin embargo, polemizando contra esta última -o desesperanzados de ella, varios críticos han supuesto que proporcionaban el golpe de gracia al análisis de clase tout court ${ }^{1}$. Por lo tanto, antes de seguir, pensamos que es importante detallar cuatro elementos en particular que el análisis de clase como nosotros lo entendemos no implica -aunque estén presentes en la mayoría de las formulaciones marxistas.

Primero, nuestra concepción del análisis de clase no implica una teoría de la historia según la cual el conflicto de clase es el motor del cambio social, de modo que, en el momento de crisis de sucesivas etapas de desarrollo, una clase particular (la clase trabajadora bajo el capitalismo) asume su "misión" de transformar la sociedad a través de la acción revolucionaria. De hecho, entre aquellos sociólogos que han estado activamente comprometidos en lo que podríamos considerar como el programa de

\footnotetext{
${ }^{1}$ En francés en el texto. Significa "sin más” (N. de T.).
} 
Goldthorpe, J. H., \& Marshall, G. (2017). El prometedor futuro del análisis de clase: Una respuesta a las críticas recientes. Revista de Sociología 32(1), 106-128. doi: 10.5354/0719-529x.2017.47888

investigación del análisis de clase, podemos encontrar una fuerte oposición a ese historicismo, ya sea de inspiración marxista o liberal (e.g. Esping-Andersen, 1990, cap. 1; Goldthorpe, 1871, 1988², 1992; Haller, 1990; Korpi, 1978; Marshall et al., 1988, cap. 10). Más bien, el énfasis está en la diversidad de los senderos de desarrollo que las naciones han seguido hacia la modernidad y en la muy variable naturaleza -porque es esencialmente contingente- del papel jugado por la formación de clases y la acción.

Segundo, el análisis de clase como lo entendemos no implica una teoría de la explotación de clase, según la cual todas las relaciones de clase deben ser necesaria y exclusivamente antagónicas, y desde la cual provendría directamente la base objetiva para una sociología y una economía "críticas". Aunque algunos exponentes del análisis de clase tal como la entendemos podrían ver desde luego el conflicto como inherente dentro de las relaciones de clase, esto no les exige adherir a una teoría del valor-trabajo, o en efecto a ninguna otra doctrina que suponga la explotación como es entendida en el discurso marxista. Tampoco deben suponer, como es sugerido por Sørensen (1991), que lo que es la ventaja de una clase debe ser siempre y completamente la desventaja de otra. En efecto, mucho del interés se ha centrado últimamente en discusiones teoréticas sobre las condiciones

\footnotetext{
2 Se corrigió un error de digitación en la cita original, en la cual figuraba el año 1979, por lo anterior, se utiliza en este artículo el año del libro en que se publica el capítulo que es 1988 (N. del Ed.).
}

bajo las cuales las relaciones de clases pueden ser mejor entendidas como un juego de sumapositiva (o suma-negativa) en vez que uno de suma-cero $^{3}$. Y este interés se ha visto reflejado mediante estudios sustantivos en una preocupación acerca del papel desempeñado por los "compromisos de clase", por ejemplo, en las relaciones laborales o en el desarrollo de políticas económicas nacionales y estados de bienestar (véase los artículos compilados en Goldthorpe, 1984).

Además, los argumentos desarrollados desde un punto de vista liberal, sea por sociólogos funcionalistas o economistas neoclásicos, respecto de que las desigualdades de clase son propicias para el bienestar mayor de todos a través de varios mecanismos, pueden ser vistos como llamados para realizar investigación empírica en vez que simples rechazos ideológicos. Y, a su vez, los resultados de tales investigaciones podrían ser considerados como relevantes para cualquier evaluación moral acerca de las desigualdades de clase. Al respecto, la influencia de las teorías marxistas de la explotación puede ser mucho menor que la del "principio de diferencia" formulado por Rawls (1972) ${ }^{4}$.

\footnotetext{
3 Estos tres conceptos (suma-positiva, suma-negativa y suma-cero) son extraídos de la teoría de juegos (positivesum, negative-sum, zero-sum), y se entienden como los resultados posibles en una disputa y/o negociación. También pueden ser traducidos como resultado-positivo, resultado-negativo y resultado-cero (N. de T.).

4 Sørensen (1991) sostiene que mientras el esquema de clases desarrollado por Goldthorpe y otros -a diferencia de los desarrollados por marxistas como Wright- no apela explícitamente a relaciones de explotación entre las diferentes clases, la idea de explotación no puede ser
} 
Goldthorpe, J. H., \& Marshall, G. (2017). El prometedor futuro del análisis de clase: Una respuesta a las críticas recientes. Revista de Sociología 32(1), 106-128. doi: 10.5354/0719-529x.2017.47888

Tercero, la versión del análisis de clase que respaldamos no toma en consideración la teoría de la acción colectiva basada en la clase, según la cual los individuos que poseen la misma posición en la estructura de clase desarrollarán por ende y automáticamente una conciencia compartida de su situación y, a su vez, se les pedirá actuar juntos para defender sus intereses comunes de clase. En efecto, conocer los nuevos desarrollos en la teoría general de la acción colectiva, desde el tiempo del estudio crucial de Olson (1965) en adelante, ha conducido a aquellos comprometidos con el análisis de clase en tanto programa de investigación a revertir eficazmente la perspectiva marxista tradicional. En vez de esperar que ocurran acciones colectivas basadas en la clase (y luego tener que recurrir a argumentos de "falsa conciencia" donde no la hay), ellos se han concentrado en establecer las

evadida por aquellos que usan el esquema, dado que el análisis de Goldthorpe asume "una teoría de la desigualdad de clase que parece necesitar un concepto de explotación en su base". Ante esto, debemos responder que Sørensen no muestra en ningún lugar por qué este argumento debería sostenerse. De hecho, el autor subsecuentemente cambia su fundamento para hacer una crítica bastante diferente: específicamente que Goldthorpe y otros no han desarrollado una teoría de la relación general entre posición de clase y recompensas diferenciadas. Podemos aceptar que este es el caso, y esta relación indudablemente requiere más investigación sistemática -incluso aunque se haya hecho desde un comienzo, más de lo que quizás Sørensen reconoce. Así, por ejemplo, la idea del "salario de eficiencia" no sólo está prefigurada en el concepto de "salario de lealtad" de Wright, como reconoce Sørensen, pero además, y más completamente, en el argumento de Renner (1953) sobre la distinción esencial entre la "relación de servicio" y el contrato de trabajo, que es desarrollado en Goldthorpe (1982) y provee un elemento central en el esquema de clase que él y sus colegas han desarrollado (cf. Erikson y Goldthorpe 1992, cap. 2). condiciones muy especiales que deben aplicarse antes de que dichas acciones puedan pensarse como probables -porque sería racional para los individuos concernidos ${ }^{-}$incluso donde los intereses compartidos de hecho son reconocidos. De este modo, cuando Pahl (1989) representa a los analistas de clase como quienes repiten irracionalmente el "mantra" "estructuraconciencia-agencia”, con los eslabones de esta cadena siendo "raramente vistos como problemáticos", esto es de hecho esencialmente lo opuesto a lo que ha pasado durante la última década o más.

En cambio, podemos añadir que los modelos de acción colectiva basada en la clase con los cuales críticos como Pahl, o Holton y Turner operan han sido en gran medida superados por trabajos recientes: es decir, el modelo revolucionario estilo "asalto al Palacio de Invierno", o el modelo gemeinschaftlich ${ }^{5}$ de la acción de la clase trabajadora basado en las solidaridades locales del lugar de trabajo o de la comunidad. $\mathrm{Si}$ tuviésemos que dar un ejemplo paradigmático de acción colectiva de clase para el análisis "post-Olson", seguramente tendríamos que hablar de las clases trabajadoras bajo las economías políticas neocorporativistas -por ejemplo, Suecia- que asume en efecto un carácter muy diferente e incluso opuesto. Esencialmente, esta acción (o algunos desearían decir inacción) corresponde a trabajadores que aceptan la participación de sus confederaciones sindicales en políticas gubernamentales de regulación salarial, y

${ }^{5}$ Comunitario (N. de T.) 
Goldthorpe, J. H., \& Marshall, G. (2017). El prometedor futuro del análisis de clase: Una respuesta a las críticas recientes. Revista de Sociología 32(1), 106-128. doi: 10.5354/0719-529x.2017.47888

aceptan mostrar una gran solidaridad de clase, absteniéndose del uso del poder de negociación localizado o seccional, de modo que sus líderes puedan perseguir de mejor manera los objetivos más generalizados de la clase trabajadora como el pleno empleo y políticas de redistribución del bienestar, como un quid pro quo ${ }^{6}$ para la moderación salarial (ver Goldthorpe, 1984: Korpi, 1983; Pizzorno, 1978; Scharpf, 1984; Stephens 1979). Entonces, desde este nuevo punto de vista, la relación conciencia-agencia es a lo menos radicalmente repensada: la conciencia de clase, citando la formulación de Elster (1985), es "la habilidad para superar el problema del free-rider en la realización de los intereses de clase" (p. 342).

Finalmente, el análisis de clase tal como lo entendemos no responde a una teoría reduccionista de la acción política -individual o colectiva- según la cual tal acción puede ser entendida simplemente como la expresión sin mediación de las relaciones de clase y la búsqueda de objetivos de clase estructuralmente determinados. En un momento en que muchos analistas de clase llegaron a un entendimiento muy cambiado de la conexión conciencia-agencia, también buscaron moverse a una nueva visión de las relaciones entre conciencia (o al menos conciencia de intereses) y estructura, nuevamente bajo la influencia de un desarrollo teorético más general (e.g. Berger, 1981). Lo que

\footnotetext{
6 "Quid pro quo" es una locución en latín que significa "una cosa por otra", refiriéndose -según la Real Academia Española- a una "cosa que sustituye a algo equivalente o que se recibe como compensación por ello" (N. de T.).
}

fue rechazado es, precisamente, la idea de que una conciencia y una preocupación acerca de los intereses de clase provengan directamente y "objetivamente" de la posición de clase. En vez de eso, la tenencia de las posiciones de clase es vista como creadora sólo de intereses potenciales, de la misma manera que puede surgir de otras posiciones estructurales. Entonces, si son los intereses de clase los que los individuos en efecto buscan realizar en vez de otros intereses dependerá en primer lugar de las identidades sociales que ellos ocupen, ya que -citando una máxima atribuida a Pizzorno- "la identidad precede al interés". Y aunque en la formación de tales identidades importan varios procesos sociales, por ejemplo, la movilidad, se enfatiza que para que los intereses de clase se conviertan en la base de la movilización política, los movimientos políticos y los partidos deben jugar un rol crucial, a través de sus ideologías, programas y estrategias (ver Esping-Andersen, 1985; Heath et al., 1991, cap. 5; Korpi 1983; Marshall et al., 1988, cap. 7; Pizzorno, 1978).

Hindess (1987, cap. 6) ha insistido, usando a algunos de los autores citados arriba, en que el análisis de clase no-marxista, al igual que el marxista, sigue aquejado de reduccionismo en su tratamiento de la política. Sin embargo, su argumento no es convincente, dado que simplemente afirma que los autores en cuestión son guiados hacia posiciones reduccionistas, sin intentar demostrar este punto, ya sea a través de citas o referencias específicas. Además, no ofrece ninguna razón por la cual los nomarxistas, que no tienen ninguna teoría de la historia en tanto lucha de clases para defender, 
Goldthorpe, J. H., \& Marshall, G. (2017). El prometedor futuro del análisis de clase: Una respuesta a las críticas recientes. Revista de Sociología 32(1), 106-128. doi: 10.5354/0719-529x.2017.47888

deberían estar atraídos por el reduccionismo o tener alguna dificultad para rechazarlo totalmente. Aunque análisis particulares pueden enfocarse en el rol que juegan los procesos sociales en vez de los procesos políticos en la formación -o descomposición- de clase, esto no implica en ningún caso que la relevancia de estos últimos sea negada. De hecho, todos los autores a los cuales Hindess se refiere han podido enfatizar la autonomía -incluso la primacía- de lo político, frente a lo que ellos considerarían como un indebido "sociologismo"7.

A la luz de estas aclaraciones, el análisis de clase en nuestro sentido bien puede aparecer como un proyecto mucho más limitado que en su versión marxista, tanto intelectual como políticamente. $\mathrm{Y}$ en efecto, en ciertos aspectos lo es, más específicamente en no derivar o ser orientada por ninguna teoría general de clase, o a su vez aspirar a formar la base de teorías aún más amplias de la sociedad o de la historia ${ }^{8}$. Sin embargo, el análisis de clase como nos gustaría defenderlo tiene ambiciones que recaen en una dirección diferente. Mientras sus partidarios pueden adherir a diferentes conceptos y teorías de clase, apuntan a ponerlos a prueba buscando problemas del tipo expuestos al comienzo de

\footnotetext{
7 Así, por ejemplo, Hindess afirma con respecto a EspingAndersen y Korpi (1984) que "los intereses de clase son considerados como objetivamente dados en la estructura de las relaciones capitalistas" (1987, p. 99). Simplemente no hay justificación para esta afirmación, y en efecto en el argumento de Esping-Andersen (1985) existe una visión diametralmente opuesta.

8 En este sentido, podríamos de hecho coincidir con las conclusiones alcanzadas por Wright (1989) respecto de proveer una comparación del análisis de clase marxista y no-marxista, desde el lado marxista.
}

este artículo y a través de la investigación que respete un estándar metodológico generalmente más adecuado para sus dificultades propias que para aquellos definidos anteriormente. Más específicamente, si en el programa de investigación del análisis de clase las preocupaciones principales consisten en examinar la importancia de la clase (y de otros factores relativos a esta) en la formación de las oportunidades de vida y los patrones de la acción social, y en rastrear cualquier cambio al respecto que pueda ocurrir en el transcurso del tiempo, entonces se debe cumplir un número de requisitos en términos de conceptualización, análisis de datos y recolección de datos. Estos tres requerimientos exigen atención aquí, tanto por su importancia sustancial como porque podrían haber sido insuficientemente apreciados por los críticos.

Primero, los conceptos de clase deben ser tan claramente definidos como sea operacionalmente factible, para evadir cualquier confusión entre clase y otros factores de posible relevancia. Holton y Turner argumentan (1989, p. 172) que "elementos de status" usualmente están presentes en el "discurso de clase", pero prestan poca atención a los esfuerzos hechos durante la última década más o menos (desde las posiciones marxistas y no-marxistas) para producir conceptos de clase y categorizaciones de un tipo analíticamente más satisfactorio. Pahl (1989) nota tales esfuerzos de parte de Wright y Goldthorpe, pero luego busca devaluarlos porque "tristemente, no parecen ser congruentes" (p. 712-713). Que este pudiera ser el caso no es, sin embargo, ni 
Goldthorpe, J. H., \& Marshall, G. (2017). El prometedor futuro del análisis de clase: Una respuesta a las críticas recientes. Revista de Sociología 32(1), 106-128. doi: 10.5354/0719-529x.2017.47888

sorprendente ni perturbador. Lo que Pahl falla en reconocer es que es precisamente una preocupación de los analistas de clase el evaluar las aproximaciones conceptuales rivales, y que hay en efecto procedimientos firmes para hacerlo (ver Marsh, 1986; Marshall, 1988; Marshall et al., 1988; Marshall \& Rose 1990). Los analistas de clase tienen un interés obvio en determinar qué categorizaciones son las más efectivas para mostrar variación de las variables dependientes bajo examen -y en parte porque quienes han deseado restar importancia a los efectos de clase han aprovechado usualmente resultados derivados de categorizaciones que son menos satisfactorias en términos tanto analíticos como empíricos. Pahl mismo (1989) provee aquí un buen ejemplo, a la vez que sugiere (1991), muy erróneamente, que la aproximación seguida no hace mucha diferencia ${ }^{9}$.

Un segundo requisito es que los análisis a realizar deben asumir que son genuinamente multivariables y que las preguntas de "textura" causal deben ser consideradas cuidadosamente. Por ejemplo, si se sostiene que el poder explicativo de la clase está disminuyendo y que ha sido superado por, digamos, diferencias en los patrones de consumo o estilos de vida, entonces tal afirmación obviamente llama a un análisis multivariable como base de su evaluación empírica. Es notable, sin embargo,

9 Para otro caso, véase Saunders (1990). Una buena ilustración del modo en el cual el uso de diferentes categorizaciones de clase puede producir resultados diferenciados en sus implicaciones sustantivas -pero también en su validez -se provee en la nota al pie número 20 de este artículo. que, aunque Pahl (1989), o Holton y Turner (1989) aborden este asunto -y, en el caso de Pahl, como si se hubiese ya definido contra el análisis de clase- ninguno toma seriamente en consideración los resultados de los estudios en los cuales se han realizado análisis multivariantes relevantes.

Nuevamente, Pahl (1989) en particular da gran importancia al argumento de que la sola demostración de que existen asociaciones entre clase y variables dependientes "está probablemente vinculando un número de procesos muy distintos que deberían mantenerse analíticamente por separado" (p. 716). Pero aquí, simplemente abre un rango de problemas con los cuales aparentemente no está muy familiarizado. Uno de ellos es cuánto podemos demostrar en cualquier caso particular -digamos, por el camino del análisis causal- que los efectos de clase están mediados por variables intervinientes. Otro problema surge respecto de la significancia teorética de los factores causales de un tipo menor y más cercano. Al contrario de lo que Pahl parece creer (1991), incluso el exitoso "desempaquetar" de la clase en la forma que él la concibe no necesariamente reduce su importancia sociológica. Así, nadie podría suponer que las causas inmediatas por ejemplo del bajo nivel educacional, de votar por los laboristas o de sufrir bronquitis crónica, son todas las mismas. Pero en tanto pueda ser rastreado el nexo de cada uno de los diferentes conjuntos de causas inmediatas involucradas con la ubicación de los individuos o las familias en (digamos) posiciones de clase trabajadora no 
especializada, entonces la importancia de la clase se incrementa en vez de disminuir. Se subraya la omnipresencia de la influencia de la clase.

Un requisito final es que los análisis de clase, y a su vez los datos con los cuales se elaboran deben incorporar de una forma $u$ otra la dimensión temporal. Tanto Pahl (1989), como Holton y Turner (1989) sostienen que la clase está perdiendo su poder explicativo como consecuencia de varias tendencias de cambio económico y social: la decadencia de la industria pesada y manufacturera y el ascenso de los servicios, el quiebre de las comunidades de la clase trabajadora "tradicional", el crecimiento del "privatismo doméstico", y así sucesivamente. Pero al hacerlo, avanzan desde algunos cambios que están razonablemente bien documentados hacia otros que no lo están; y en cuanto a los efectos de clase per se, no hacen referencia en absoluto a hallazgos obtenidos en base a análisis de cohorte o estudios longitudinales o de panel del tipo que serían necesarios para dar a su posición un respaldo empírico adecuado. En vez de eso, podría decirse que proveen una buena ilustración de "la tendencia hacia el pensamiento histórico dual" contra la cual Marshall et al. (1988) han alertado explícitamente: es decir, una tendencia "a través de la cual un proletariado comunitario y solidario de algunas ya pasadas épocas de oro del antagonismo de clase es levantado contra la clase trabajadora de hoy atomizada y orientada al consumo" (p. 206) -de manera que sin embargo, esto tenga poco sustento tanto en investigaciones sociológicas como históricas.

\section{Algunos resultados ilustrativos}

En esta segunda parte de nuestro artículo, llamamos la atención, aunque en una forma muy resumida, sobre los hallazgos de tres áreas dentro del programa de investigación del análisis de clase que, podríamos argumentar, cualquier crítico seria debiera considerar -y especialmente si su último objetivo consiste en establecer que el análisis de clases ya no juega un rol útil en el estudio de las sociedades modernas. Discutiremos la movilidad de clase, la clase y la educación y la clase y la pertenencia partidista.

\section{Movilidad de clase}

Para estudiar la movilidad social dentro del contexto de la estructura de clase, en vez de elegir una jerarquía de status, por ejemplo, debemos tomar una decisión conceptual a priori (Goldthorpe, 1985). No obstante, cuando se asume esta perspectiva, los resultados no han sido de poca importancia sociológica.

Para los propósitos presentes, podemos destacar que, a lo largo de parámetros nacionales diversos, se ha usado las clases para visualizar "características de movilidad" bastante diversas: es decir, en una perspectiva de entrada, se han visto en la homogeneidad de los orígenes de clase de aquellos individuos que componen su membresía actual; y en una perspectiva de salida, en el poder de retención o la "capacidad de agarre", ambos respecto de los tiempos de vida tanto individuales como inter- 
Goldthorpe, J. H., \& Marshall, G. (2017). El prometedor futuro del análisis de clase: Una respuesta a las críticas recientes. Revista de Sociología 32(1), 106-128. doi: 10.5354/0719-529x.2017.47888

generacionales (Erikson \& Goldthrope, 1992; Featherman \& Selbee, 1988; Featherman, Selbee, \& Mayer, 1989; Marshall et al., 1988; Mayer et al., 1989). Así, por ejemplo, las clases de servicio o los asalariados de las sociedades modernas tienden a ser altamente heterogéneos en su composición, pero también tienden a tener un gran poder de retención tanto desde el punto de vista intra- como inter-generacional. En comparación, las clases trabajadoras son más homogéneas en su composición, y las clases agrarias muchísimo más, pero ambas clases revelan bajo poder de retención, especialmente en términos inter-generacionales. En otras clases, tales como en la pequeña burguesía y los empleados de rutina no-manuales, las combinaciones de homogeneidad, vida laboral y poder de retención intergeneracional son también diferentes.

Se puede demostrar que tales características de movilidad tienen un origen doble. Primero, reflejan el hecho de que las clases -definidas en términos de relaciones de empleo entre diferentes sectores de las economías nacionalestienden a seguir trayectorias bastante peculiares, o "historias naturales" de crecimiento o disminución en relación con el desarrollo estructural de estas economías (de un modo en el que los estratos definidos en términos de status o prestigio no lo hacen) ${ }^{10}$. Y

\footnotetext{
${ }^{10}$ Las escalas de prestigio o de status tienden a juntar muy adecuadamente en sus propios términosagrupaciones laborales o de otro tipo que tienen lugares muy dispares dentro de los mercados del trabajo, las unidades de producción y los sectores económicos; y estas agrupaciones tienen probablemente trayectorias muy diferentes de crecimiento o declive (véase Westergaard y
}

segundo, reflejan el hecho de que las diferentes clases tienden a ser asociadas con "propensiones" específicas hacia la inmovilidad o la movilidad independientemente de todos los efectos estructurales. Cabe notar que este último hallazgo es posible sólo gracias a avances técnicos en el análisis de tablas de movilidad, que han permitido graficar una distinción conceptual crucial entre tasas "absolutas" y "relativas" (véase Goldthorpe, 1980, 1987; Hauser, 1978; Hauser et al., 1975).

Que las clases puedan mostrar tales diferencias en las características de movilidad puede entonces sugerir en sí mismo que son capaces de ser definidas de una manera que no es más meramente arbitraria, y que los "problemas de los límites" que algunos críticos han destacado son en buena parte más manejables que lo que ellos tratan de implicar. Ciertamente, se puede cuestionar las bases de afirmaciones tales como las hechas por Holton y Turner (1989), de que es "muy difícil combinar la multiplicidad de posiciones de clase en categorías, sin recurrir a criterios evaluativos culturales" (p. 174) ${ }^{11}$.

Además, es en términos de tales características de movilidad que la formación de clase puede

Resler 1975, p. 287; Erikson y Goldthorpe 1992, cap. 6). Así, donde la jerarquía de prestigio o de status sea tomada como contexto conceptual para el estudio de la movilidad, se mermará probablemente el alcance total de los efectos derivados del cambio estructural en la economía.

11 Nótese que nosotros aquí no estamos argumentando a favor del procedimiento, defendido por Brieger (1981) y otros como esencialmente "weberiano", en el cual los límites de clase son realmente determinados sobre la base del análisis de movilidad. Si esta aproximación puede o no encontrar algún respaldo serio en el trabajo de Weber es en nuestra opinión excesivamente empirista y posiblemente llevaría a problemas interpretativos mayores. 
Goldthorpe, J. H., \& Marshall, G. (2017). El prometedor futuro del análisis de clase: Una respuesta a las críticas recientes. Revista de Sociología 32(1), 106-128. doi: 10.5354/0719-529x.2017.47888

ser evaluada en su nivel "demográfico" básico (Goldthorpe, 1980, 1987); es decir, en términos del alcance y la naturaleza de la asociación que existe entre individuos o familias y posiciones particulares de clase a través del tiempo. Y esto a su vez puede ser visto como un determinante del potencial de las clases en tanto colectividades, para desarrollar también subculturas distintivas y una "capacidad de socialización”, que son a su vez los prerrequisitos clave para que las identidades de clase sean creadas (Featherman \& Spenner, 1990)12. En otras palabras, aquí se ofrece un enfoque en el que trabajamos activamente para investigar procesos de formación o descomposición de clase a través de indagaciones empíricas sistemáticas. No se supone, tal como lo hace el marxismo dogmático, que la formación de clase está de alguna forma programada históricamente. Pero no asumimos tampoco en la manera en que lo hacen Pahl, o de Holton y Turner que en las sociedades modernas la descomposición de clase es un fenómeno ampliamente generalizado. Y tal como hemos indicado, la evidencia producida hasta ahora apunta de hecho hacia la existencia de una situación mucho más compleja.

\footnotetext{
12 Para repetir, sin embargo, siempre se debe reconocer la importancia de los factores políticos en estos procesos. Nótese además que las características de movilidad a las cuales referimos aquí son definidas en términos de tasas absolutas (aunque las tasas relativas juegan por supuesto un papel en su determinación).
}

Clase y educación

La educación es la fuerza compensatoria más frecuentemente citada en los argumentos que afirman que la influencia de la clase en las oportunidades de vida individuales está disminuyendo. De acuerdo con aquellas teorías de la sociedad industrial que plantean, en términos de Holton y Turner, "el desafío del liberalismo" al análisis de clase, la "lógica" misma del industrialismo requiere que tanto la oferta como el acceso a la educación se amplíen constantemente, y además que el logro educacional se convierta en la clave determinante del éxito en la vida económica. Entonces, se espera a su vez que la asociación entre orígenes de clase y logro educacional se debilite, mientras que aquella entre logro educacional y destinos de clase se fortalezca, y en sí medie (y legitime) la mayoría de las asociaciones entre orígenes y destinos de clase que puedan seguir existiendo (e.g. Blau \& Duncan, 1967; Kerr, 1983; Kerr et al., 1960; Treiman, 1970). En otras palabras, existe un progresivo tránsito desde una sociedad de clases "cerrada" hacia una sociedad meritocrática de un tipo supuestamente más "abierto".

Sin embargo, a la luz de los resultados de investigaciones acumulados hasta ahora, este escenario liberal no es impresionante. Cambios de largo plazo en las interrelaciones entre clase y educación del tipo señalado son difícilmente detectables en la mayoría de las sociedades a nivel nacional (véase especialmente Blossfeld \& Shavit, 1992). Además, sólidos hallazgos transnacionales plantean otro problema mayor 
Goldthorpe, J. H., \& Marshall, G. (2017). El prometedor futuro del análisis de clase: Una respuesta a las críticas recientes. Revista de Sociología 32(1), 106-128. doi: 10.5354/0719-529x.2017.47888

en el ámbito de la investigación sobre movilidad: a saber, que las tasas relativas de movilidad de clase intergeneracional muestran en general un alto grado de estabilidad temporal (Erikson y Goldthorpe, 1992). Por ejemplo, en el caso de Gran Bretaña, al menos cuatro análisis independientes han revelado poco cambio en estas tasas durante el curso del presente siglo -y ciertamente ninguno en la dirección de una mayor fluidez (Goldthorpe, 1980, 1987; Hope, 1981; Macdonald \& Ridge, 1987; Marshall et al., 1988). Así, incluso si pudiese establecerse que la selección social se ha vuelto más meritocrática, existen pocos indicios de que esto tenga algún efecto en producir más oportunidades equitativas en cuanto a la movilidad de clase.

En el caso británico, donde la investigación en este tema ha sido quizás más amplia que en ningún otro lugar, Halsey (1977) sugirió inicialmente que a pesar de que existía alguna evidencia respecto de un "vínculo estrecho"13 entre educación y logro laboral a la mitad del siglo, esto había sido compensado por crecientes diferencias de clase en el logro educacional, acompañado por una baja o nula reducción en la fuerza de los efectos "directos" (aquellos que no son mediados vía educación) de los orígenes de clase sobre el destino de clase. A la luz de las investigaciones posteriores basadas en datos longitudinales más amplios y técnicas analíticas más refinadas, parece difícil defender la existencia de un real crecimiento de las

13 Traducido del inglés "tightening bond" que puede ser entendido a su vez como "vínculo estrecho" o "vínculo endurecido" (N. de T.). diferencias de clase en el ámbito educacional; y el debate se ha volcado más bien hacia si estas diferencias se han mantenido esencialmente inalteradas o han mostrado un grado de estrechamiento en algunos aspectos (Heath \& Clifford, 1990; Jonsson \& Mills, 1991) ${ }^{14}$. Pero lo que también surge es una duda mayor acerca de la presunta tendencia secular del logro educacional de volverse más importante en tanto determinante de la clase de destino. El incremento de la selección ocupacional por mérito, al menos en la medida que este es determinado por credenciales educacionales, no es fácil de discernir (véase Heath, Mills, \& Roberts, 1991; Jonsson, 1991, para resultados similares para Suecia).

En suma, la evidencia a favor de la educación que operaría como una fuerza de "disminución de clase"15 sigue siendo leve. En cambio, las investigaciones a las cuales nos hemos referido sugieren que es esperable un alto grado de resistencia a cualquier tendencia que favorezca una reducción de las desigualdades de clase vía "meritocracia". Si la educación se convirtiera en algo más importante para determinar las oportunidades de vida laboral, entonces los miembros de las clases relativamente más aventajadas buscarían usar sus recursos para asegurar que sus niños mantuvieran una

\footnotetext{
14 Mucho parece girar aquí (y también en debates análogos en los Estados Unidos) en torno a cómo se mide el logro educacional: esto es, simplemente, en referencia al número de años de educación o a través de clasificaciones más o menos refinadas de carreras educacionales o de cualificaciones obtenidas.

15 Traducido del inglés "class abatement" que puede ser entendido también como "mitigación de clase" o "reducción de clase" (N. de T.).
} 
Goldthorpe, J. H., \& Marshall, G. (2017). El prometedor futuro del análisis de clase: Una respuesta a las críticas recientes. Revista de Sociología 32(1), 106-128. doi: 10.5354/0719-529x.2017.47888

ventaja competitiva en el logro educacional; o como lo formula Halsey (1977,), "las fuerzas adscriptivas encuentran formas de expresarse como "logro"' (p. 184). Alternativamente, y como parece quizás el hecho más probable, si las diferencias de clase en el logro educacional han disminuido hasta cierto punto, entonces entre las clases más aventajadas, los recursos familiares pueden ser aplicados a través de otros canales, con el fin de ayudar a sus hijos a preservar sus perspectivas de clase contra la amenaza de la selección meritocrática (ver Marshall \& Swift, 1992). Nos gustaría enfatizar que no queremos argumentar aquí que las desigualdades de clase no pueden nunca ser mitigadas a través de cambios en los sistemas educacionales y su funcionamiento: sólo queremos señalar que no hay razón para suponer, como lo desean los teóricos liberales, que esto probablemente ocurra como el resultado automático y favorable de los procesos sociales que se dan en el desarrollo de las sociedades industriales ${ }^{16}$.

Clase y partidismo político

Para quienes creen que en las sociedades modernas el impacto de la clase en las

\footnotetext{
16 Incluso entre quienes siguen creyendo que se puede mostrar alguna asociación entre industrialismo y crecimiento igualitario en el logro educacional y las oportunidades de movilidad relativa, su relación ahora es considerada como derivada no de la necesidad de desarrollo, sino como el resultado más contingente de una variedad de factores, incluyendo los políticos. Véase por ejemplo Treiman y Yip (1989), Ganzeboom, Luijkx y Treiman (1989). Se puede encontrar comentarios críticos sobre los datos y los análisis de estos estudios en Müller y Karle (1990), Jones (1991) y Erikson y Goldthorpe (1992).
}

oportunidades de vida está en declive, existe una progresión natural hacia la afirmación adicional de que la clase también ha perdido importancia en determinar la respuesta de los individuos a su situación social, en particular a través de la acción política. Durante los años 1950 y 1960, los sociólogos liberales estaban complacidos de describir la participación de los ciudadanos en las políticas electorales de las naciones occidentales como una representación de "la traducción democrática de la lucha de clases" (Lipset, 1960). Sin embargo, bajo la influencia tanto de los desarrollos políticos como sociales de finales de 1970 en adelante, se adoptó una posición mucho más fuerte. Ahora se sostenía que la clase estaba disolviéndose (finalmente) en cuanto base del partidismo político, y esto era más evidente en el apoyo decreciente de la clase trabajadora a los partidos de izquierda (e.g. Clark \& Lipset, 1991; Lipset, 1981). Además, este diagnóstico ha sido aceptado por muchos líderes antiguos de tipo social marxisant ${ }^{17}$, en su $\operatorname{adieux}^{18}$ desesperado a la clase trabajadora en particular y al análisis de clase en general.

En el caso británico, la tesis de "desalineación de clase"19 en la política partidista tiene quizás

17 En francés en el texto, neologismo. Significa "marxizante" (N. de T.).

18 En francés en el texto. Significa "adiós" o "despedida" (N. de T.).

19 Traducido de "Class dealignment". Este término puede ser entendido también como "desvinculación de clase", "abandono de clase" o "desajuste de clase". "Dealignment" no tiene una traducción directa al español y aquí se toma como un antónimo de "Realignment", que se traduce como "realineación". "Class dealignment" hace referencia al proceso en el cual miembros de una clase ya no se 
Goldthorpe, J. H., \& Marshall, G. (2017). El prometedor futuro del análisis de clase: Una respuesta a las críticas recientes. Revista de Sociología 32(1), 106-128. doi: 10.5354/0719-529x.2017.47888

una historia más larga que en cualquier otro lugar, y luego de los triunfos electorales de los conservadores de 1979 y 1983 fue entusiastamente revivida por una serie de autores (e.g. Butler \& Kavanagh, 1984; Crewe, 1984; Franklin, 1985; Robertson, 1984; Rose \& McAllister 1986)20. Entre los últimos, se creía que la contraparte al efecto decreciente de la clase sobre el voto era una tendencia de los conflictos políticos de partido a organizarse alrededor de "temas" más que alrededor de "intereses" socialmente estructurados. Sin embargo, en opinión de otros comentaristas, la clase estaba cediendo frente a nuevas fracturas estructurales como la base del apoyo de partido -en particular, clivajes que separaban a individuos y familias, considerados tanto como productores o consumidores, según su posición en el sector público o privado de la economía (véase Duke \& Edgell, 1984; Dunleavy, 1979, 1980; Dunleavy \& Husbands, 1985; Saunders, 1984).

Los críticos del análisis de clase tales como Pahl, o Holton y Turner aparentemente han mirado poco más allá de este rango de literatura. Escriben como si la tesis de la desalineación de clase estuviera fuertemente establecida dentro de la sociología electoral y

consideran parte de esta, desalineándose de la misma (N. de T.).

20 El énfasis central estaba puesto por supuesto en los factores que tendían a reducir el apoyo por el Partido Laborista entre la clase trabajadora -tal como en el período posterior a la tercera derrota electoral sucesiva sufrida por los laboristas en 1959. Sin embargo, la versión de 1970 de la "desalineación de clase" se concentraba más bien en las causas de la disminución del apoyo de la clase media hacia los conservadores. como si el "nuevo estructuralismo" ahora proporcionara el paradigma a tener en cuenta (Holton \& Turner, 1989; Pahl, 1989). Sin embargo, lo que fallan totalmente en reconocer es el punto en el cual tanto el "nuevo estructuralismo" como la afirmación subyacente de la desalineación de clase han sido cuestionados empíricamente y sobre la base de investigaciones y análisis que han elevado significativamente los estándares técnicos en el campo.

Más importante aún, Heath y sus colegas han mostrado la necesidad de introducir en el debate sobre la desalineación una distinción entre tasas absolutas y relativas respecto del voto de clase, análoga a aquella entre tasas absolutas y relativas de movilidad social (Evans, Heath, \& Paine 1991; Heath, Jowell, \& Curtice, 1985; Heath et al., 1991). Al aplicar esta distinción a los datos sobre votaciones en las elecciones británicas desde 1964 a 1987, muestran que los cambios en los patrones absolutos de votación de clase (o, esto es, realmente observada) son casi totalmente atribuibles a dos factores: por un lado, a cambios en la "forma" de la estructura de clase, sobre todo el crecimiento de la clase de servicio o asalariada y la disminución en tamaño de la clase trabajadora industrial; y por otra parte, a cambios en el número de partidos políticos disputando elecciones $\mathrm{y}$ en su efectividad general (esto es, su capacidad para ganar respaldo "transversalmente en el ajedrez político", en igual grado de miembros de todas las clases). En contraste, los cambios en la votación relativa de clase -o, en otras palabras, 
Goldthorpe, J. H., \& Marshall, G. (2017). El prometedor futuro del análisis de clase: Una respuesta a las críticas recientes. Revista de Sociología 32(1), 106-128. doi: 10.5354/0719-529x.2017.47888

en la asociación neta entre membresía de clase y votación- resultan ser bastante leves. Además, en la medida que tales cambios pueden ser detectados, no muestran una tendencia secular de disminución en la asociación clase-voto, y parecen más abiertos a una explicación en términos políticos en vez que sociológicos (véase especialmente Heath et al., 1991). Aunque para algunos estos hallazgos han resultado ser inquietantemente contra-intuitivos, es importante notar que están confirmados en sus fundamentos por otros análisis bastante independientes, aunque más restringidos (ver Hibbs, 1982; Marshall et al., 1988; Weakliem, 1989) ${ }^{21}$.

Mientras Heath y sus colegas continúan argumentando, sus resultados resaltan claramente los peligros del "pensamiento histórico dual" en el problema de la formación de clase, en el cual críticos del análisis de clase han tendido a participar. Hasta ahora al menos en relación con el partidismo político, no hay razón de hecho para suponer que durante las

${ }^{21}$ Las críticas del trabajo de Heath y sus colegas sobre la asociación clase-voto (e.g. Crewe 1987; Dunleavy 1987) no han sido impresionantes, por decir lo menos, y han recibido poca atención (Heath, Jowell \& Curtice 1987a, 1987b). Algunos comentarios posteriores en el debate (e.g. Edgell \& Duke 1991, p. 55-58) muestran una inquietante falta de comprensión de los procedimientos seguidos por Heath y sus colegas, así como de los asuntos involucrados. También es perturbador el continuo uso del "Índice de Alford" como una medida de "votación de clase" como por ejemplo en Clark y Lipset (1991, fig. 1), cuando las graves deficiencias de éste han sido conocidas por mucho tiempo (e.g. Korpi 1983, p. 97-99; Heath, Jowell \& Curtice 1985, p. 40-41). En contra de los resultados reportados por Clark y Lipset se pueden presentar los de Weakliem (1991), que derivan de análisis muchos más apropiados y muestran que una asociación clase-voto más o menos estable no es de ningún modo una peculiaridad británica. décadas recientes las clases sociales en Gran Bretaña -incluyendo a la clase trabajadorahayan mostrado algún debilitamiento, tanto en su cohesión social como en su carácter ideológico. Esta conclusión también es consistente con una variedad de otros hallazgos por ejemplo en tendencias (o ausencia de éstas) respecto de los patrones de movilidad de clase, respecto de los niveles de identificación de clase, y respecto de las diferencias de clase relacionadas con actitudes políticas y valores (Heath, 1990; Heath et al., 1991)22.

Además, al mismo tiempo que la tesis de la desalineación de clase ha sido cuestionada, también lo han sido las afirmaciones del "nuevo estructuralismo", al menos en sus versiones más ambiciosas. Es importante reconocer aquí que el argumento de que el partidismo político puede estar influenciado, sobre y por debajo de los efectos de clase, por factores tales como si un individuo está empleado en el sector público o

22 Lo que fue considerado -al nivel del diario sociológico dominical- como el ejemplo más obvio de la descomposición de la clase trabajadora fue la propensión distintiva de los "trabajadores cualificados" de abandonar a los laboristas por los conservadores. Sin embargo, la evidencia citada para apoyar esta afirmación podría ser un producto de la composición cambiante de la categoría MRS "C2". Aunque usualmente se refiere a esta como la de los trabajadores cualificados, la categoría es en términos de clase bastante heterogénea, incluyendo en adición a los trabajadores asalariados de base, supervisores manuales y artesanos auto-empleados. Estas últimas agrupaciones siempre han tenido una mayor tasa de votación conservadora que las primeras y, desde finales de 1970 en adelante, pueden haber sido un factor de crecimiento de la categoría en su conjunto. Si se adopta una clase trabajadora cualificada definida más adecuadamente, no emerge ninguna tendencia clara respecto de que sus miembros sean más propensos que los miembros de otras clases de votar por los conservadores en vez de los laboristas (véase Heath, Jowell, \& Curtice 1986). 
Goldthorpe, J. H., \& Marshall, G. (2017). El prometedor futuro del análisis de clase: Una respuesta a las críticas recientes. Revista de Sociología 32(1), 106-128. doi: 10.5354/0719-529x.2017.47888

privado, es propietario de su casa o arrendatario, es en sí mismo bastante antiguo y, en cualquier caso, no crea problema alguno para los exponentes del análisis de clase. Estos últimos nunca han supuesto que la clase por sí sola determina el voto; y las fuentes de diferenciación respecto de las orientaciones políticas y de la acción dentro de las clases han sido siempre de interés para ellos. El análisis de clase sólo es cuestionado en la medida en que afirma que los clivajes sectoriales han superado por ahora aquellos de clase en proveer la base estructural principal de partidismo a lo largo del electorado. Sin embargo, es exactamente este tipo de argumento el que ha sido socavado empíricamente en los estudios citados más arriba (véase especialmente Heath et al., 1991; también Marshall et al., 1988).

Así, por ejemplo, respecto de los clivajes "de producción”, se puede ver algún efecto sobre el voto en el asalariado según si los individuos están empleados en el sector privado o en diferentes ramas del sector público -aunque es probable que esto al menos refleje en parte más factores ocupacionales específicos y también procesos de auto-selección. Pero para los propósitos aquí presentes, el punto importante es que no se encuentran efectos comparables dentro de la clase trabajadora. En cambio, con relación a clivajes "de consumo" que se traducen en la tenencia de vivienda ${ }^{23}$, se puede ver algún efecto sobre el voto en la clase trabajadora -

\footnotetext{
23 Traducido del inglés "Housing tenure", que puede ser entendido también como "tipo de tenencia de vivienda", y se asocia a los acuerdos bajo los cuales una persona tiene derecho a vivir en una casa (N. de T.).
}

aunque cuestionando nuevamente la dirección de la influencia causal- pero tal efecto aparece dentro del asalariado.

En otras palabras, existen dos características muy diferentes de clivajes sectoriales, siendo que ninguno resulta ejercer una influencia en el partidismo o tener la generalidad o la fuerza global de la influencia de clase. Recientemente, se pudo observar que el peso de la evidencia empírica parecería haber llevado a algunos defensores del clivaje sectorial a modificar posiciones que adoptaron previamente. Así por ejemplo, Saunders (1990) afirma que su más reciente investigación "parece confirmar estudios previos que afirman que la significación electoral de la tenencia de vivienda es secundaria respecto de la clase social y que sus efectos pueden ser más pronunciados en algunos estratos... que en otros" (p. 234).24

Desde el punto de vista del programa de investigación del análisis de clase, la debilidad relativa de los efectos sectoriales no es de hecho difícil de entender, especialmente en relación al

24 Cuando desarrolla análisis multivariados apropiados, Saunders (1990) de hecho no puede detectar ningún efecto significativo de la tenencia de vivienda en el voto dentro de su (muy pequeña) muestra. Argumenta que algunos efectos más específicos están indicados dentro de las clases "intermedias" colapsadas en el esquema Goldthorpe. Sin embargo, es extremadamente desacertado realizar este colapso en cualquier análisis respecto del voto, ya que los patrones de votación de los miembros de estas clases son muy diferentes. Nuevamente, Edgell y Duke (1991, p. 69), después de tomar el "nuevo estructuralismo" con gran seriedad, deben reconocer que "la desalineación de clase es un mito", y lo que más pueden decir para los factores sectoriales es que influencian el voto de una elección a otra "dependiendo de las circunstancias históricas particulares". Además, incluso esta conclusión no resulta convincente desde los análisis que ellos reportan, los cuales son muy inadecuados para los temas que abordan. 
Goldthorpe, J. H., \& Marshall, G. (2017). El prometedor futuro del análisis de clase: Una respuesta a las críticas recientes. Revista de Sociología 32(1), 106-128. doi: 10.5354/0719-529x.2017.47888

consumo. Por una parte, los "problemas de límite" aquí son verdaderamente formidables, ya que muchos, si no la mayoría de los individuos, estarán ampliamente involucrados tanto en el sector público como en el sector privado simultáneamente. Por otro lado, los regímenes de movilidad determinan el grado de asociación en el tiempo entre individuos o familias, y las diferentes posiciones sectoriales parecerían ser mucho más fluidas que aquellas que aplican en el caso de la clase. ${ }^{25}$ Así, se puede señalar que las dudas que Pahl, o Holton y Turner expresan respecto a la formación de identidades colectivas y respecto de una comunidad percibida de intereses sobre la base de la clase deberían aplicar a fortiori ${ }^{26}$ en la medida que los clivajes de consumo estén involucrados -aunque esto es de hecho un punto que ellos pasan por alto.

\section{CONCLUSIONES}

Hemos buscado en este artículo responder a las críticas recientes del análisis de clase en dos áreas principales. Primero, afirmamos que los críticos no han distinguido adecuadamente entre el análisis de clase en sus versiones marxistas y el análisis de clase como programa de investigación. Varias objeciones

\footnotetext{
25 Que esto en efecto es así con la vivienda, ha sido mostrado por Savage, Watt y Arber (1990) para un área del Sud-Este de Inglaterra, y sus resultados son confirmados por análisis preliminares de datos a nivel nacional actualmente realizados por Maireád Reidy en Nuffield College, Oxford.

26 "A fortiori" es una locución latina que, según la RAE, significa “con mayor razón” (N. de T).
}

poderosamente planteadas contra el primero simplemente no aplican para el segundo. Esto no es muy sorprendente, dado que el análisis de clase visto como un campo de investigación sociológica empírica liberado de enredos con la filosofía de la historia y la "teoría crítica" se desarrolló de hecho como una reacción contra el marxismo. Segundo, hemos intentado mostrar, en referencia a tres tópicos centrales, que el programa de investigación del análisis de clase ha producido resultados, permitiendo rechazar rotundamente las afirmaciones de Pahl, o de Holton y Turner de que la clase como un concepto ya no es útil, y conserva sólo un valor retórico y no científico. ${ }^{27}$

Finalmente, podemos notar que las dos líneas principales de argumentación que hemos seguido convergen en cierto modo. Para los marxistas, el análisis de clase era la clave para comprender el cambio social a largo plazo: las relaciones de clase y específicamente el conflicto de clase proporcionaban el motor de este cambio, y el estudio de sus dinámicas era crucial para alcanzar la comprensión cognitiva deseada respecto del movimiento de la historia. Sin embargo, el análisis de clase en tanto programa de investigación no sólo es una empresa intelectual de un tipo muy diferente al del análisis de clase del marxismo, sino que también genera resultados que dan una nueva perspectiva sobre la significación sustantiva de las relaciones de clase en la sociedad

\footnotetext{
27 En esta conexión vale la pena notar que hemos citado una veintena de libros, monografías y documentos de investigación recientes, ejemplificando el análisis de clase tal como nosotros lo entendemos, el cual, por alguna razón, virtualmente no recibió ninguna mención de estos críticos.
} 
Goldthorpe, J. H., \& Marshall, G. (2017). El prometedor futuro del análisis de clase: Una respuesta a las críticas recientes. Revista de Sociología 32(1), 106-128. doi: 10.5354/0719-529x.2017.47888

contemporánea. Un tema común en los hallazgos ahora acumulados de las investigaciones es, como hemos visto, aquel de la estabilidad en vez del dinamismo de las relaciones de clase. Aparece una notable persistencia de las desigualdades vinculadas a la clase y de los patrones de acción social diferenciados por clase, incluso en períodos de cambio acelerado a nivel de la estructura económica, las instituciones sociales y las coyunturas políticas. La revelación de tal estabilidad -hecha posible en gran medida por los avances en las técnicas de análisis de datos y la construcción de conjuntos de datos que mencionamos- parece a su vez traer dos implicaciones importantes. Obviamente, estos problemas son creados por los teóricos liberales de la sociedad industrial que podrían anticipar el más o menos espontáneo "desvanecimiento" de la clase, y asimismo del análisis de clase. Pero al mismo tiempo, existe la necesidad de reorientar radicalmente las inquietudes teóricas de los defensores del análisis de clase. Deben enfocarse no en la explicación del cambio social vía relaciones de clase, sino en entender los procesos que subyacen a la profunda resistencia al cambio que tales relaciones presentan.

\section{Reconocimientos}

Los autores desean agradecer a Geoff Evans, A.H. Halsey y Ray Pahl por sus útiles comentarios en un borrador previo de este artículo.

\section{REFERENCIAS}

Bauman, Z. (1982). Memories of class. London: Routledge and Kegan Paul.

Berger, S. (1981). Organizing interests in western Europe. Cambridge: Cambridge University Press.

Blau, P. M., \& Duncan, O. D. (1967). The American occupational structure. New York: Wiley.

Blossfeld, H., \& Shavit, Y. (1992). Persistent inequality: Changing educational stratification in thirteen countries. Boulder, Colarado: Westview Press.
Breiger, R. (1981). The social class structure of occupational mobility. American Journal of Sociology, 87, 578-591.

Butler, D., \& Kavanagh, D. (1985). The British general election of 1983. London: Macmillan.

Clark, T., \& Lipset, S. M. (1991). Are social classes dying?. International Sociology, 6, 397-410.

Crewe, I. (1984). The electorate: Partisan dealignment ten years On. En H. Berrington (Ed.), Change in British politics. London: Frank Cass. 
Goldthorpe, J. H., \& Marshall, G. (2017). El prometedor futuro del análisis de clase: Una respuesta a las críticas recientes. Revista de Sociología 32(1), 106-128. doi: 10.5354/0719-529x.2017.47888

Crewe, I. (1987). On the death and resurrection of class voting: some comments on how britain votes. Political Studies, 34, 620-638.

Duke, V., \& Edgell, S. (1984). Public expenditure cuts in britain and consumption sectoral cleavages. International Journal of Urban and Regional Research, 8, 177-201.

Dunleavy, P. (1979). The urban bases of political alignment: social class, domestic property ownership, and state intervention in consumption processes. British Journal of Political Science, 9, 403-443.

Dunleavy, P. (1980). The political implications of sectoral cleavages and the growth of state employment. Political Studies, 28, 364-383.

Dunleavy, P. (1987). Class dealignment in Britain revisited: Why odds ratios give odd results. West European Politics, 10, 400-419.

Dunleavy, P., \& Husbands, C. T. (1985). British democracy at the crossroads. London: George Allen and Unwin.

Edgell, S., \& Duke, V. (1991). Measure of thatcherism. London: Harper Collins.

Elster, J. (1985). Making sense of Marx. Cambridge: Cambridge University Press.

Erikson, R., \& Goldthorpe, J. H. (1992). The constant flux: Class mobility in industrial societies. Oxford: Clarendon Press.

Esping-Andersen, G. (1985). Politics against markets. Princeton: Princeton University Press.

Esping-Andersen, G. (1990). The three worlds of welfare capitalism. Cambridge: Polity Press.

Esping-Andersen, G., \& Korpi, W. (1984). Social policy as class politics in post-war capitalism: Scandinavia, Austria and Germany. En J. H.
Goldthorpe (Ed.), Order and conflict in contemporary capitalism. Oxford: Clarendon Press.

Evans, G., Heath, A., \& Payne, C. (1991). Modelling trends in the class/party relationship 1964-87. Electoral Studies, 10, 99-117.

Featherman, D. L., Selbee, L. K. (1988). Class formation and class mobility: A new approach with counts from life history data. En M. Riley, \& B. Huber (Eds.), Social structure and human lives. Newbury Park: Sage.

Featherman, D.L., Spenner, K. I. (1990). Class and the socialisation of children: Constancy, change or irrelevance? En E. M. Hetherington, R. M. Lerner, \& M. Perlmutter (Eds.), Child development in life-span perspective. Hillsdale, New Jersey: Erlbaum.

Featherman, D., Selbee, L. K., \& Mayer, K. U. (1989). Social class and the structuring of the life course in Norway and West Germany. En D. Kertzer, J. Meyer, \& K. W. Schale (Eds.), Social structure and aging. Hillsdale, New Jersey: Erlbaum.

Franklin, M. N. (1985). The decline of class voting in Britain. Oxford: Clarendon Press. Ganzeboom, H., Luijkx, R. \& Treiman, D. J. (1989). Intergenerational class mobility in comparative perspective. Research in Social Stratification and Mobility, 8, 3-55.

Goldthorpe, J. H. (1971). Theories of industrial society: reflections on the recrudescence of historicism and the future of futurology. Archives Euopéennes de Sociologie, 12, 263288. 
Goldthorpe, J. H., \& Marshall, G. (2017). El prometedor futuro del análisis de clase: Una respuesta a las críticas recientes. Revista de Sociología 32(1), 106-128. doi: 10.5354/0719-529x.2017.47888

Goldthorpe, J. H. (1988). Intellectuals and the Working Class in Modern Britain', Fuller Bequest Lecture, David Rose (Ed.), Social Stratification and Economic Change. London: Hutchinson.

Goldthorpe, J. H., Llewellyn, C., \& Payne, C. (1980). Social mobility and class structure in modern Britain. Oxford: Clarendon Press.

Goldthorpe, J. H. (1982). On the service class: Its formation and future. En A. Giddens \& G. Mackenzie (Eds.), Social class and the division of labour. Cambridge: Cambridge University Press.

Goldthorpe, J. H. (1984). The end of convergence: Corporatist and dualist tendencies. In modern western societies. En J. H. Goldthorpe (Ed.), Order and conflict in contemporary capitalism. Oxford: Clarendon Press.

Goldthorpe, J. H. (1985). Soziale Mobilität und klassenbildung. zur erneuerung einer tradition soziologischer Forschung. En H. Strasser \& J. H. Goldthorpe (Eds.), Die analyse sozialer ungleichheit. Opladen: Westdeutcher Verlag.

Goldthorpe, J. H. (1992). Employment, class and mobility: A critique of liberal and marxist theories of long term change. En $\mathrm{H}$. Haferkamp \& N. Smelser (Eds.), Modernity and social change. Berkeley: University of California Press.

Gorz, A. (1982). Farewell to the working class. London: Pluto Press.

Haller, M. (1990). European class structure: does it exist?. En M. Haller (Ed.), Class structure in Europe: new findings from east- west comparisons of social structure and mobility. London: M. E. Sharpe.

Halsey, A. H. (1977). Towards meritocracy? The case of Britain. En A. H. Halsey \& J. Karabel (Eds.), Power and ideology in education. New York: Oxford University Press.

Hauser, R. M. (1978). A structural model of the mobility table. Social Forces, 56, 919-953.

Hauser, R. M., Dickinson, P. J., Travis, H. P. \& Koffel, J. M. (1975). Temporal change in occupational mobility: Evidence for men in the united states. American Sociological Review, 40, 279-297.

Heath, A. (1990). Class and political partisanship. En J. Clark, C. Modgil \& S. Modgil (Eds.), John H. Goldthorpe: Consensus and controversy. London: Falmer.

Heath, A., \& Clifford, P. (1990). Class inequalities in education in the twentieth century. Journal of the Royal Statistical Society, Series A, 153, 1-16.

Heath, A., Jowell, R., \& Curtice, J. (1985). How Britain Votes. Oxford: Pergamon.

Heath, A., Jowell, R., \& Curtice, J. (1986). Understanding electoral change in Britain. Parliamentary Affairs, 39, 150-164.

Heath, A., Jowell, R., \& Curtice, J. (1987a). Trendless fluctuation: a reply to crewe. Political Studies, 35, 256-277.

Heath, A., Jowell, R., \& Curtice, J. (1987b). Class dealignment and the explanation of political change: a reply to dunleavy. West European Politics, 11, 146-148.

Heath, A., Mills, C., \& Roberts, J. (1991). Towards meritocracy? Recent evidence on an old problem. Joint unit for the study of social 
Goldthorpe, J. H., \& Marshall, G. (2017). El prometedor futuro del análisis de clase: Una respuesta a las críticas recientes. Revista de Sociología 32(1), 106-128. doi: 10.5354/0719-529x.2017.47888

trends, SCPR-Nuffield College, Oxford Working Paper 3.

Heath, A., et al. (1991). Understanding Political Change: The British Voter, 1964-1987. Oxford: Pergamon.

Hibbs, D. (1982). Economic outcomes and political support for British governments among the occupational classes. American Political Science Review, 76, 259-79.

Hindess, B. (1987). Politics and class analysis. Oxford: Basil Blackwell.

Hobsbawm, E. (1981). The forward march of labour halted? and observations on the debate. En M. Jacques \& F. Mulhern (Eds.), The forward march of labour halted? London: New Left Books.

Holton, R. J., \& Turner, B. S. (1989). Has class analysis a future? Max Weber and the challenge of liberalism to gemeinschaftlich accounts of class. R. J. Holton \& B. S. Turner (Eds.), Max Weber on economy and society. London: Routledge and Kegan Paul.

Hope, K. (1981). Trends in the openness of British society in the present century. Research in Social Stratification and Mobility, 1, 127-169.

Jones, F. L. (1991). Common social fluidity: A comment on some recent criticism. European Sociological Review, 8(3), 233-237.

Jonsson, J. O. (1991a). Towards the meritselective society? Swedish Institute for Social Research.

Jonsson, J. O. (1991b.). Class formation: The holding power and socio-demographic composition of social classes in Sweden. Swedish Institute for Social Research.
Jonsson, J. O., \& Mills, C. (1991). Social class and educational attainment in historical perspective: A Swedish-English comparison. Swedish Institute for Social Research.

Kerr, C. (1983). The future of industrial society. Cambridge, Massachusetts: Harvard University Press.

Kerr, C., Dunlop, J., Harbison, F., \& Myers, C. (1960). Industrialism and industrial man. Cambridge, Massachusetts: Harvard University Press.

Korpi, W. (1978). The working class in welfare capitalism: Work, unions and politics in Sweden. London: Routledge.

Korpi, W. (1983). The democratic class struggle. London: Routledge.

Lakatos, I. (1970). Falsification and the methodology of scientific research programmes. En I. Lakatos \& A. Musgrave (Eds.), Criticism and the growth of knowledge. Cambridge: Cambridge University Press.

Lipset, S. M. (1960). Political man. London: Heinemann.

Lipset, S. M. (1981). Whatever happened to the proletariat?. Encounter, 56, 18-34.

Lukes, S. (1984). The future of British socialism?. En B. Pimlott (Ed.), Fabian essays in socialist thought. London: Heinemann.

Macdonald, K., \& Ridge, J. (1987). Social mobility. En A. H. Halsey (Ed.), Trends in British society since 1900 (2a ed.). London: Macmillan. 
Goldthorpe, J. H., \& Marshall, G. (2017). El prometedor futuro del análisis de clase: Una respuesta a las críticas recientes. Revista de Sociología 32(1), 106-128. doi: 10.5354/0719-529x.2017.47888

Marsh, C. (1986). Occupationally-based measures of social class. London: Social Research Association.

Marshall, G. (1988). Classes in britain: official and marxist. European Sociological Review, 4, 141-154.

Marshall, G., \& Rose, D. (1990). Out-classed by our critics?. Sociology, 24, 255-67.

Marshall, G., \& Swift, A. (1992). Social class and social justice. British Journal of Sociology, 44(2), 187-211.

Marshall, G., Newby, H., Rose, D., \& Vogler, C. (1988). Class in modern Britain. London: Hutchinson.

Mayer, K. U., Featherman, D., Selbee, L. K., \& Colbjornsen, T. (1989). Class mobility during working life: A cross-national comparison of Germany and Norway. En M. Kohn (Ed.), Cross-national research in sociology. Newbury Park: Sage.

Mills, C. W. (1959). The sociological imagination. Oxford: Oxford University Press.

Müller, W., \& Karle, W. (1990). Social Selection in Educational Systems in Europe. Trabajo presentado para la ISA Research Committee on Social Stratification and Mobility, Madrid.

Offe, C. (1985). 'Work: the Key Sociological Category?' in his Disorganized Capitalism. Cambridge: Polity Press.

Olson, M. (1965). The logic of collective action. Cambridge, Massacnusetts: Harvard University Press.

Pahl, R. E. (1989). Is the emperor naked? Some comments on the adequacy of sociological theory in urban and regional research.
International Journal of Urban and Regional Research, 13, 709-720.

Pahl, R. E. (1991). R. E. Pahl replies. International Journal of Urban and Regional Research, 15, 127-129.

Pizzorno, A. (1978). Political exchange and collective identity in industrial conflict. En C. Crouch \& A. Pizzorno (Eds.), The resurgence of class conflict in Western Europe since 1968 (Vol. 2). London: Macmillan.

Rawls, J. (1972). A theory of justice. Oxford: Clarendon Press.

Renner, K. (1953). Wandlungen der modernen gesellschaft: Zwei abhandlungen über die probleme der nachkriegszeit. Vienna: Wiener Volksbuchhandlung.

Robertson, D. (1984). Class and the British electorate. Oxford: Blackwell.

Rose, R., \& Mcallister, I. (1986). Voters begin to choose. London: Sage.

Saunders, P. (1984). Beyond housing classes: the sociological significance of private property rights in means of consumption. International Journal of Urban and Regional Research, 8, 202-227.

Saunders, P. (1990). A nation of home owners. London: Unwin Hyman.

Savage, M., Watt, P., \& Arber, S. (1990). The consumption sector debate and housing mobility. Sociology, 24, 97-117.

Scharpf, F. W. (1984). Economic and institutional constraints on full employment strategies: Sweden, Austria and West Germany, 1973-1982. En Goldthorpe (Ed.), Order and conflict in contemporary capitalism. New York: Clarendon Press 
Goldthorpe, J. H., \& Marshall, G. (2017). El prometedor futuro del análisis de clase: Una respuesta a las críticas recientes. Revista de Sociología 32(1), 106-128. doi: 10.5354/0719-529x.2017.47888

Sørensen, A. B. (1991). On the usefulness of class analysis in research on social mobility and socioeconomic inequality. Acta Sociologica, 34, 71-87.

Stephens, J. D. (1979). The transition from capitalism to socialism. London: Macmillan. Treiman, D. J. (1970). Industrialism and social stratification. En E. O. Laumann (Ed.), Social stratification: Research and theory for the 1970s. New York: Bobbs-Merril.

Treiman, D. J., \& Yip, K. (1989). Educational and occupational attainment in 21 countries. En Kohn (Ed.), Cross-national research in sociology. Newbury Park: Sage.
Weakliem, D. (1989). Class and party in Britain, 1964-1983. Sociology, 23, 285-297.

Weakliem, D. (1991). The two lefts? Occupation and party choice in France, Italy, and the Netherlands. American Journal of Sociology, 96, 1327-1361.

Westergaard, J. H., \& Resler, H. (1975). Class in a capitalist society. Harmonds worth: Penguin.

Wright, E. O. (1989). Rethinking, once again, the concept of class structure. En E. O. Wright et al. The debate on classes. London: Verso. 